\title{
LOCAL HEATING IN THE GALACTIC CENTER WESTERN ARC
}

\author{
N. Mariñas, C. M. Telesco, ${ }^{1}$ R. K. Piña, ${ }^{1}$ R. S. Fisher, ${ }^{1,2}$ and M. C. Wyatt ${ }^{1,3}$ \\ Department of Astronomy, University of Florida, Gainesville, FL 32611 \\ Received 2002 May 6; accepted 2002 November 13
}

\begin{abstract}
We present arcsecond resolution, 10.8, 11.7, and $18.2 \mu \mathrm{m}$ images of the central few parsecs of the Galaxy. These images show for the first time the clumpiness of the dust in the western arc. The 11.7 and $18.2 \mu \mathrm{m}$ images of part of the western arc were used to evaluate the dust temperature and optical depth distribution of this region. We see several mid-infrared emission peaks that coincide with dust temperature peaks and regions of low optical depth, and we infer the presence of embedded sources in the western arc. Minimum luminosity estimates for two of these sources $\left(5 \times 10^{4} L_{\odot}\right.$ and $\left.2 \times 10^{4} L_{\odot}\right)$ suggest that the circumnuclear ring is being locally heated by relatively young stars.

Key words: dust, extinction — Galaxy: center — Galaxy: stellar content — infrared radiation
\end{abstract}

\section{INTRODUCTION}

The western arc is a feature several parsecs long that has been interpreted as the ionized inner edge of the Galactic center molecular ring (Lacy, Achtermann, \& Serabyn 1991; Telesco, Davidson, \& Werner 1996; Latvakoski et al. 1999). Along with the northern arm, the eastern arm, and the bar, all of which are identified with ionization fronts, the western arc dominates the appearance of the central few parsecs of the Galaxy at mid-infrared wavelengths. These morphological features are identified in Figure 1.

Infrared mapping of the central parsec of the Sgr A West region has revealed peaks of emission (Rieke, Telesco, \& Harper 1978; Aitken, Allen, \& Roche 1982; Rieke \& Lebofsky 1982; Gezari et al. 1985). If these sources coincide with peaks in the dust temperature, they could be heated either by embedded, possibly recently formed stars that have not cleared the dust around them (Gezari et al. 1985), or by radiation generated externally. This issue can be resolved if the dust density distribution is also known. High-density clumps heated by an external source can have their temperatures elevated by Ly $\alpha$ heating. As a result of resonant scattering of Ly $\alpha$ photons, dust grains in areas of high density can experience an intense $\operatorname{Ly} \alpha$ radiation field that raises the temperature of the clump above that of the surrounding areas without there being a luminosity source within the clump (Gatley 1982). On the other hand, if the temperature peaks coincide with low-density regions, embedded stars that have partially cleared the dust around them are probably the ionizing sources (Gezari, Dwek, \& Varosi 1994).

Large-scale mapping of the central $5 \mathrm{pc}$ of the Galaxy at 10,20 , and $30 \mu \mathrm{m}$ at $4^{\prime \prime}$ angular resolution by Telesco et al. (1996) found a temperature gradient consistent with most of the heating throughout the central few parsecs of the galaxy being powered by luminous sources located within the central parsec. Studies at 32 and $38 \mu \mathrm{m}$ corroborate these

\footnotetext{
${ }^{1}$ Visiting Astronomer at the IRTF, which is operated by the University of Hawaii under a cooperative agreement with the National Aeronautics and Space Administration.

${ }^{2}$ Now at the Gemini Observatory, Hilo, HI 96720.

${ }^{3}$ Now at the UK Astronomy Technology Centre, Royal Observatory, Blackford Hill, Edinburgh, UK.
}

findings (Latvakoski et al. 1999). A comparison of the infrared images with thermal radio continuum $(2.6 \mathrm{~cm})$ and molecular tracers $(\mathrm{HCN})$ shows a progression of ionized, photodissociated, and molecular material away from the cluster center, with the mid-infrared emission coinciding with the ionized inner edge of the circumnuclear ring (CNR) as expected from central heating. However, modeling the Galactic center dust as optically thin silicate grains heated by the UV radiation from the central star cluster cannot reproduce the high dust temperatures observed at some locations in the CNR (Telesco et al. 1996; Chan et al. 1997). Chan et al. calculated a maximum temperature of $94 \mathrm{~K}$ at the inner edge of the CNR using these models, while some observed values are in excess of $150 \mathrm{~K}$. These findings suggest that additional local heating sources contribute to the mid- and far-infrared emission of the CNR.

Gezari et al. (1994) imaged the central parsec of the Galaxy at a resolution of 1"11 at wavelengths 4.8-12.4 and $20 \mu \mathrm{m}$ and found that the positions of mid-infrared sources correlate with local temperature peaks and regions of low dust density. A comparison of temperature maps with the positions of the He I star cluster members imaged by Krabbe et al. (1991) at $2.06 \mu \mathrm{m}$ showed temperature enhancements at the stars' positions. These studies indicate that some regions in the central parsec are being heated by the $\mathrm{He} \mathrm{I}$ emission-line star cluster and by other embedded luminous stars. These sources can serve as archetypes for local heating in the CNR because they illustrate the correlation between identified embedded stars, elevated dust temperature, and low dust density.

In this paper we present multiwavelength mid-IR images of the western arc that bear on the issue of local heating of the CNR. Our observations provide new information about the distribution of the color temperature and optical depth structure of this region. We show that the presence of relatively young embedded stars may contribute significantly to the heating of the CNR.

\section{OBSERVATIONS}

We imaged the Galactic center on 1996 May 16 and 17 and on 1997 September 8 using OSCIR, the University of Florida mid-infrared imager/spectrometer camera, at the 


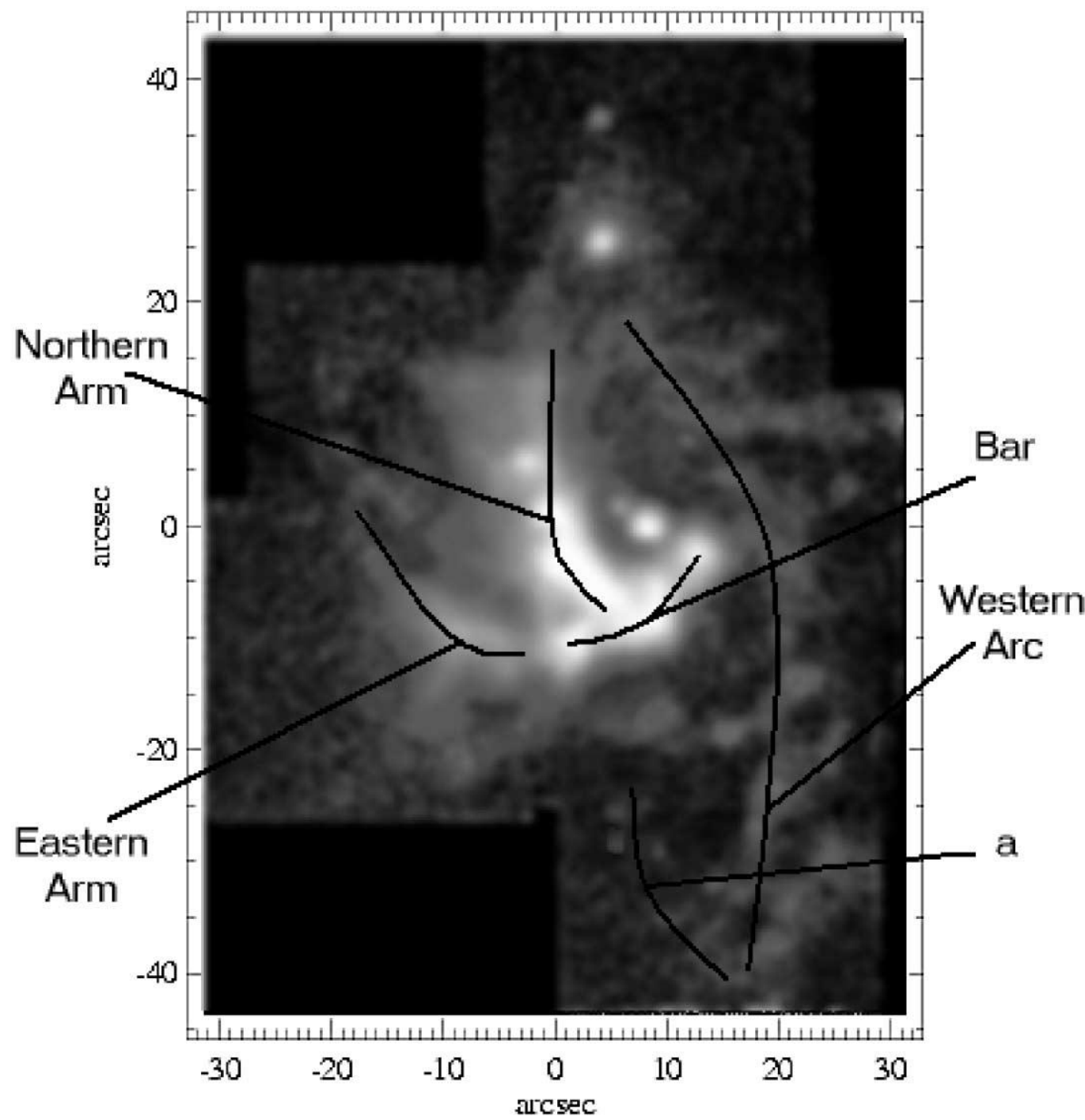

FIG. 1.-Large-scale $10.8 \mu \mathrm{m}$ OSCIR image of the GC, with inset showing location of clumpy region studied in more detail. The region labeled "a" corresponds to southeastern region of the CNR. The source IRS 1 is located at the origin $(0,0)$.

NASA Infrared Telescope Facility (IRTF). OSCIR contains a $128 \times 128$ pixel Si:As HF-16 block impurity band (BIB) Rockwell/Boeing detector. When used at the IRTF, the pixel scale is 0 ".223 and the camera field of view subtends $28^{\prime \prime} \times 28^{\prime \prime}$. For this observation the chop/nod mode was

TABLE 1

STANDARd Stars Assumed Flux DENSITIES

\begin{tabular}{ccc}
\hline \hline Star & $\begin{array}{c}\text { Filter } \\
(\mu \mathrm{m})\end{array}$ & $\begin{array}{c}\text { Flux Density } \\
(\mathrm{Jy})\end{array}$ \\
\hline$\alpha$ Her ............ & 10.8 & 1197 \\
& 11.7 & 1040 \\
& 18.2 & 458.2 \\
$\alpha$ Sco ............. & 10.8 & 2158 \\
& 11.7 & 1870 \\
$\gamma$ Aql............. & 18.2 & 815.1 \\
& 10.8 & 71.4 \\
& 11.7 & 61.1 \\
& 18.2 & 25.7 \\
\hline
\end{tabular}

used, with a total integration time of 1 minute per field position. An area of $60^{\prime \prime} \times 80^{\prime \prime}$ centered on IRS 1 was imaged in the $N$ band $(10.8 \mu \mathrm{m}, \Delta \lambda=5.23 \mu \mathrm{m})$ to obtain a mosaic of the region, including the CNR. To study the morphology of the western arc, images at $10.8 \mu \mathrm{m}(\Delta \lambda=5.23 \mu \mathrm{m}), 11.7 \mu \mathrm{m}$ $(\Delta \lambda=1.11 \mu \mathrm{m})$, and $18.2 \mu \mathrm{m}(\Delta \lambda=1.65 \mu \mathrm{m})$ were taken, spanning an area $29^{\prime \prime} \times 29^{\prime \prime}$ centered $20^{\prime \prime}$ west, $29^{\prime \prime}$ south of IRS 1 . All the images were processed using the interactive data language (IDL). The mid-infrared stars $\alpha$ Her, $\alpha$ Sco, and $\gamma$ Aql were used for flux calibration and air-mass correction. Assumed flux densities for the standards are listed in Table 1. The star SAO 209696 was used to obtain the point-spread function (PSF) required to smooth images of the western arc to the same resolution before obtaining the color temperature and optical depth maps; however, during these observations we were seeing-limited, with a typical FWHM for the PSF of 1"!2.

\section{RESULTS}

The large-scale $N$-band mosaic shown in Figure 1 spans a region $60^{\prime \prime} \times 80^{\prime \prime}$, which corresponds to $2.5 \mathrm{pc} \times 3.3 \mathrm{pc}$. The 
coordinate system of the image is centered on the IRS 1 emission peak. The map reveals the clumpy morphology of the dust emission throughout the region and presents the first detection of the faint southeastern portion of the CNR in the $10 \mu \mathrm{m}$ region, labeled " a " in Figure 1. The brightest emission peak in this region was detected at $189 \mathrm{mJy}$ with a $6 \sigma$ significance. This region was first detected in the infrared by Latvakoski et al. 1999 at 31 and $37 \mu \mathrm{m}$. Figure $2 a$ shows the southwestern region of the CNR at $10.8 \mu \mathrm{m}$. Dust emission peaks are resolved with source sizes of $2^{\prime \prime}$ to $5^{\prime \prime}\left(1.7 \times 10^{4}\right.$ to $4.3 \times 10^{4} \mathrm{AU}$ ). Scans of the brighter emission peaks and PSF star at $10.8 \mu \mathrm{m}$ are shown on Figures $2 b$ and $2 c$.

We assessed the dust temperature and optical depth for the southwestern region using the 11.7 and $18.2 \mu \mathrm{m}$ images, since this combination is less affected by the silicate feature spanning the $10 \mu \mathrm{m}$ region. Images were cross-convolved to the same resolution (the $11.7 \mu \mathrm{m}$ image was convolved with the $18.2 \mu \mathrm{m}$ PSF, and the $18.2 \mu \mathrm{m}$ image was convolved with the $11.7 \mu \mathrm{m}$ PSF) and registered, so that peak fluxes in the images were located at the same positions. This was possible because with our resolution we see no difference in the structure of the region at 11.7 and $18.2 \mu \mathrm{m}$. The observed intensity from each pixel was modeled as arising from an isothermal source by using the standard relation

$$
F_{\nu}=\Omega\left(1-e^{-\tau}\right) B_{\nu}(T) e^{-\tau_{\mathrm{ISM}}},
$$

where $\Omega$ is the solid angle, $\tau(\nu) \propto \lambda^{-1}$ is the opacity of the emitting dust, $\tau_{\text {ISM }}$ is the interstellar extinction, and $B_{\nu}(T)$ is Planck function at dust temperature $T$. We estimate a uniform $\tau_{\text {ISM }}$ across our 11.7 and $18.2 \mu \mathrm{m}$ passbands of 2.86 and 2.20 , respectively, by using the interstellar extinction

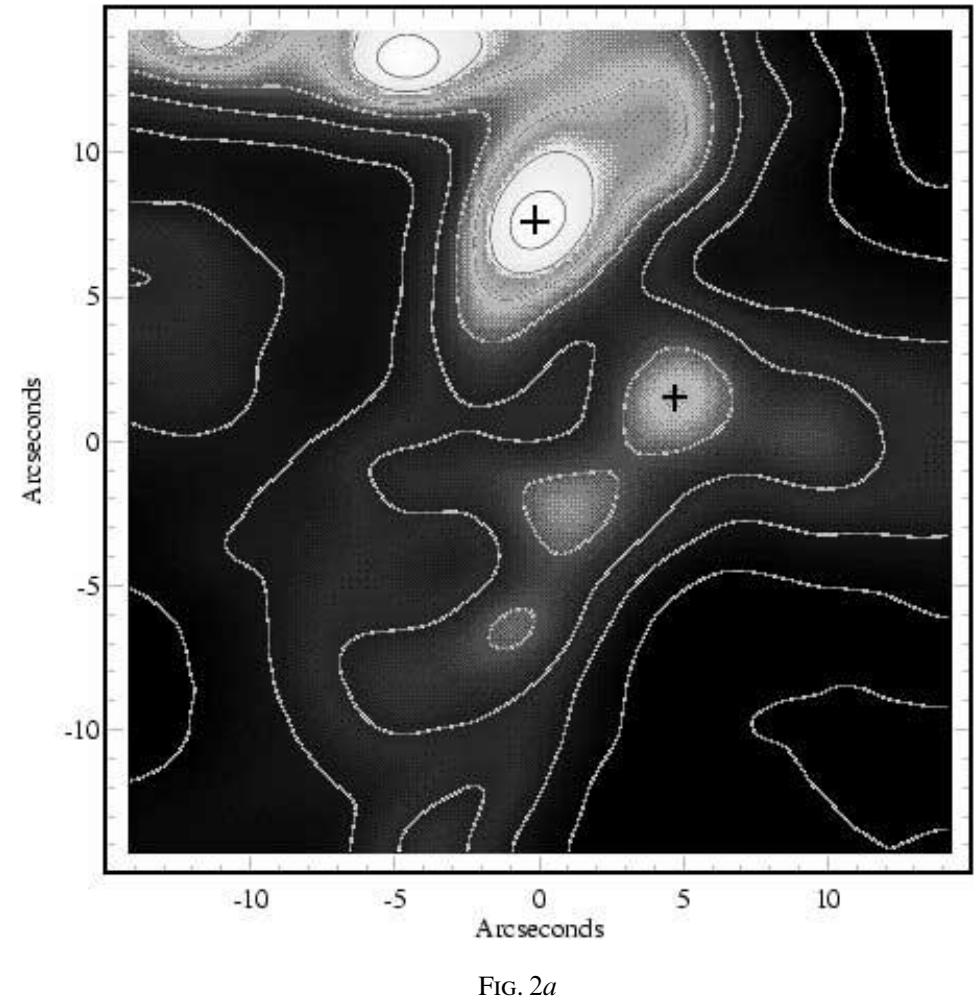

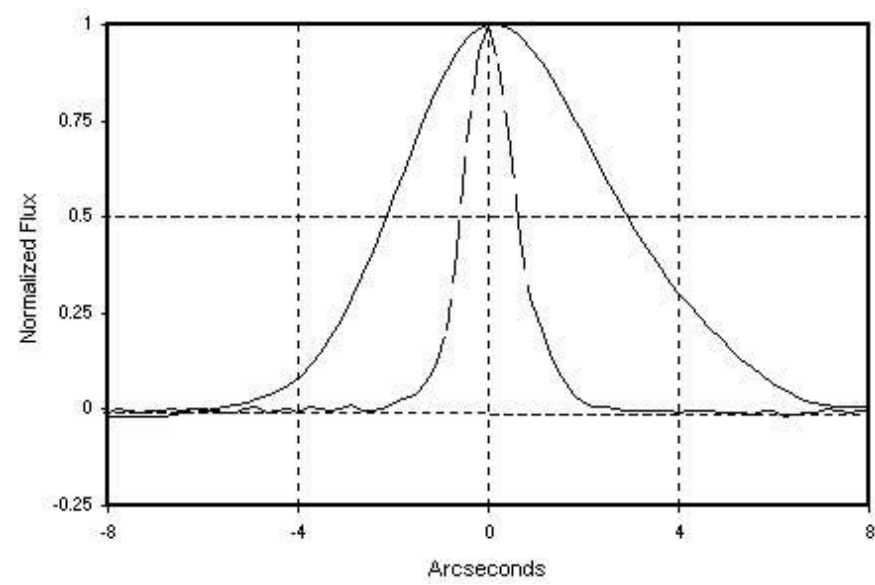

FIG. $2 b$

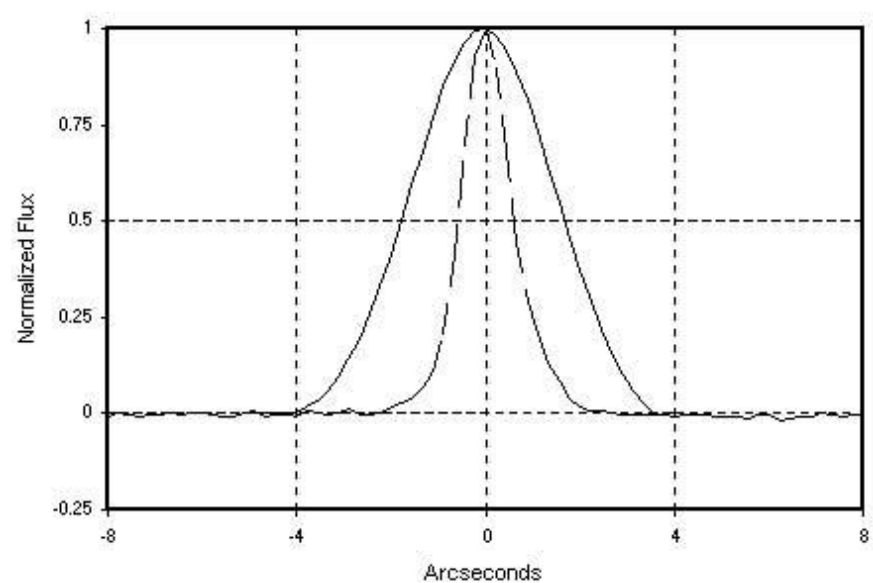

FIG. $2 c$

FIG. 2.- (a) $10.8 \mu$ m contour emission map of a region of the western arc centered $20^{\prime \prime}$ west, $29^{\prime \prime}$ south of Sgr 1 . Crosses mark emission peaks studied in more detail. Contour levels are 0.00, 1.25, 2.50, 3.75, 5.00, 6.25, 7.50, $8.75 \mathrm{mJy} \mathrm{pixel}^{-1}$. (b) Scans in R.A. of source 1 (solid line) and PSF star at $10.8 \mu \mathrm{m}($ dashed line). (c) Scan in R.A. of source 2 (solid line) and PSF star at $10.8 \mu \mathrm{m}$ (dashed line). 


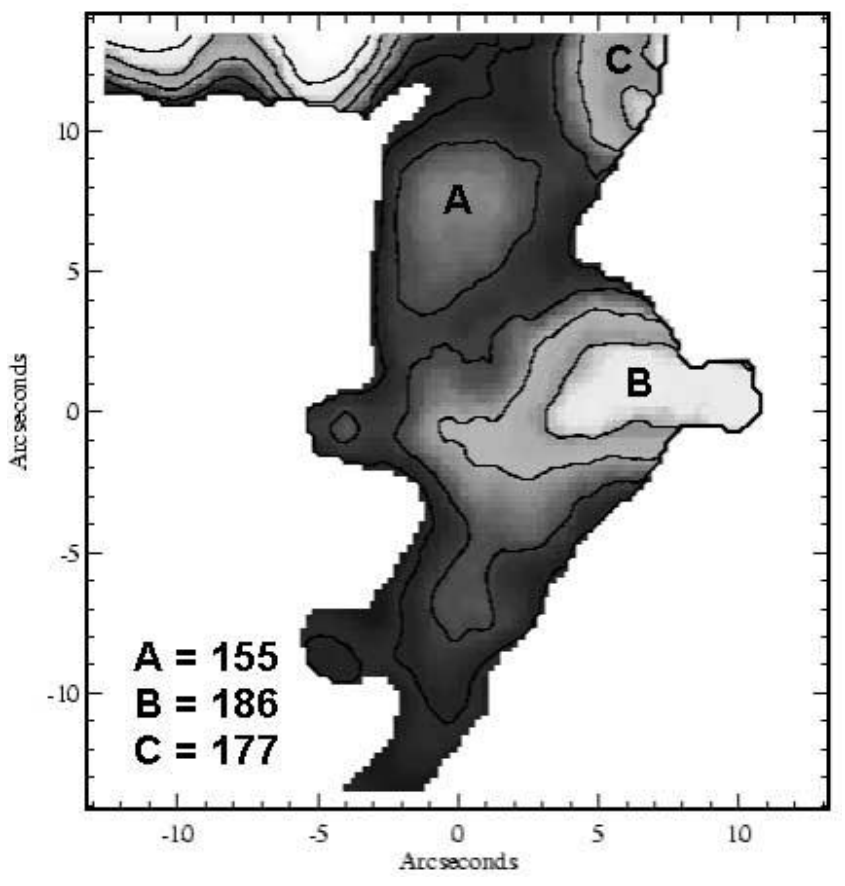

FIG. $3 a$

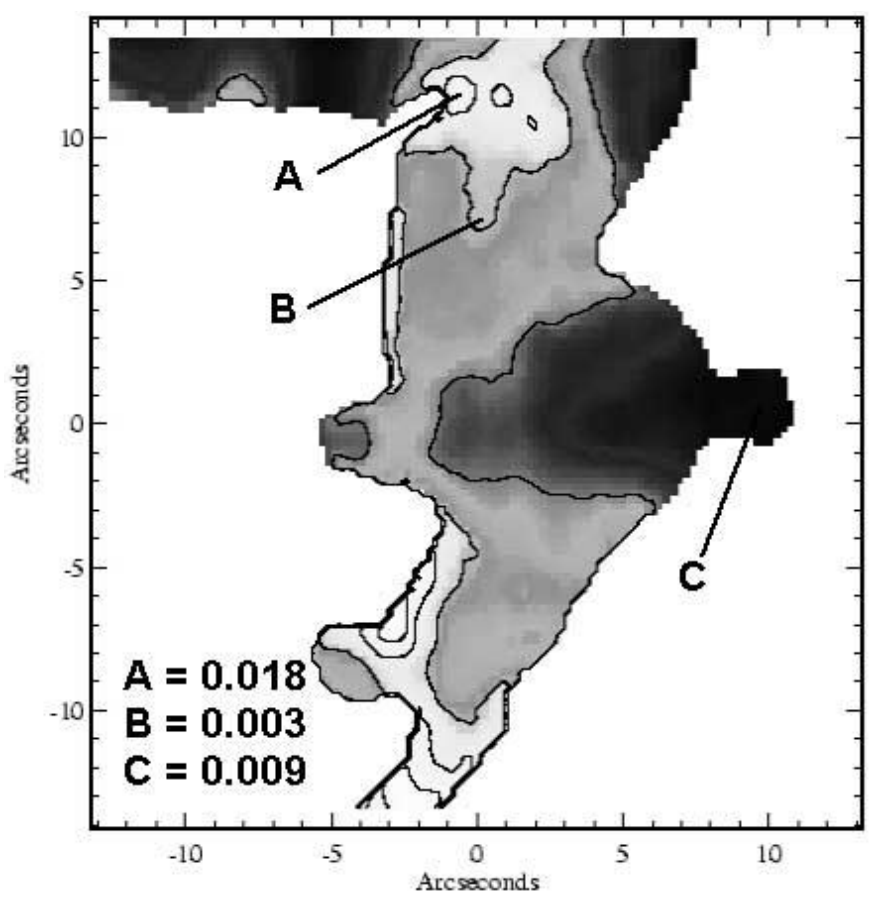

FIG. $3 b$

FIG. 3.- (a) 11.7/18.2 $\mu \mathrm{m}$ color temperature map. Contour levels are 130, 145, 160, 175, 190, $205 \mathrm{~K}(T)$. (b) 11.7/18.2 $\mu \mathrm{m}$ optical depth map $(\tau)$. Contour levels are $0.005,0.01,0.015,0.02$. Light color indicates high values of temperature (left) and opacity (right).

curve from Mathis (1990) with a value of 3.1 for the ratio of total-to-selective extinction $\left(R_{V}\right)$ and a value of 2.1 for $\tau_{\text {ISM }}$ at $18.7 \mu \mathrm{m}$ (McCarthy et al. 1980). Figure $3 a$ shows the resulting color temperature map, and Figure $3 b$ shows the corresponding optical depth map. The maps only show regions for which the signal-to-noise ratio in the flux ratio of the 11.7 and $18.2 \mu \mathrm{m}$ images is greater than 3 .

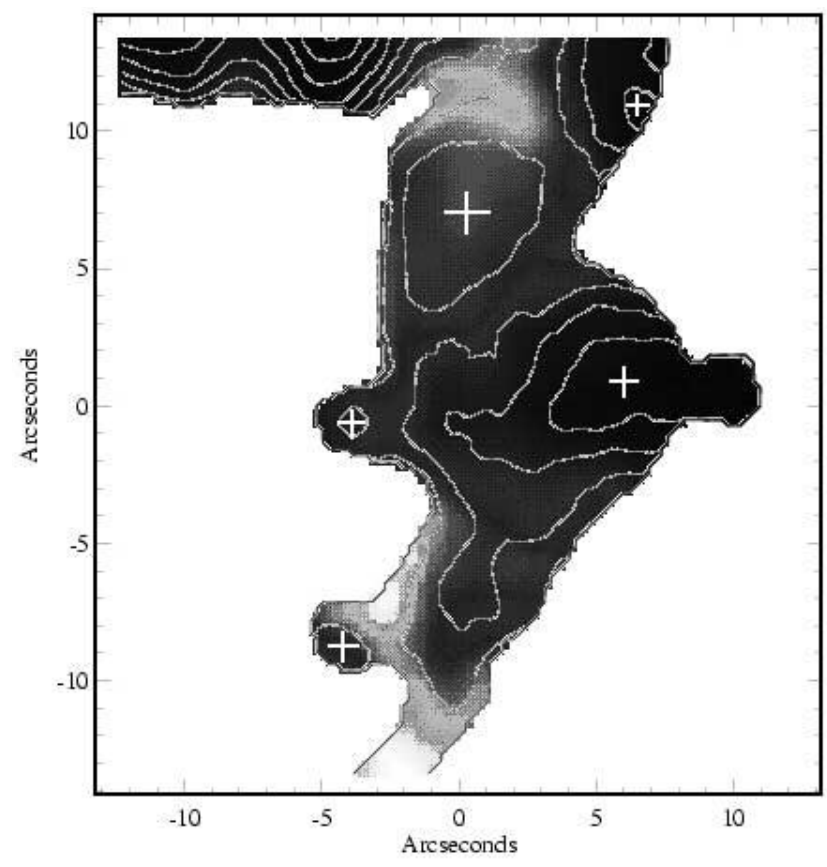

FIG. $4 a$
The temperature map shows a similar clumpy structure to that evident in the brightness map, and at this resolution we do not see the temperature decline with distance from the center observed at lower angular resolution. When comparing the temperature and optical depth distribution map, we see that the temperature peaks are located in areas of low optical depth. Figure $4 a$ shows the color temperature

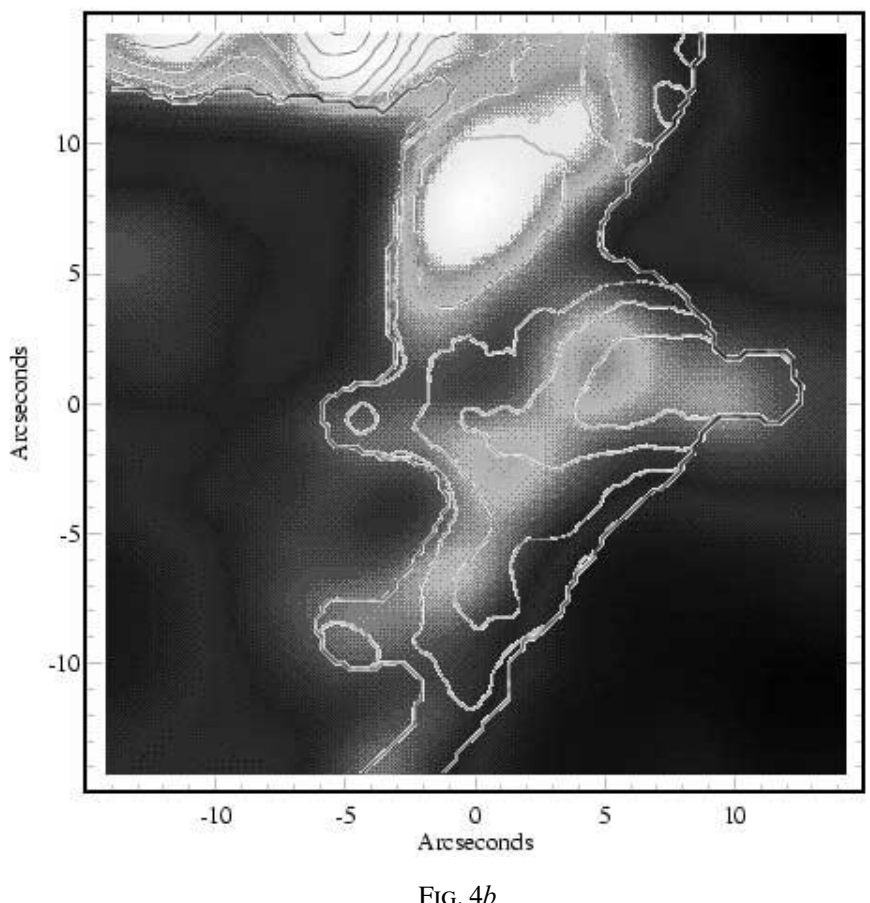

FIG. 4.- (a) Temperature contour map on our optical depth map (light color indicates high opacity; crosses mark temperature peaks). (b) Temperature contour map on the 10.8 brightness map. 


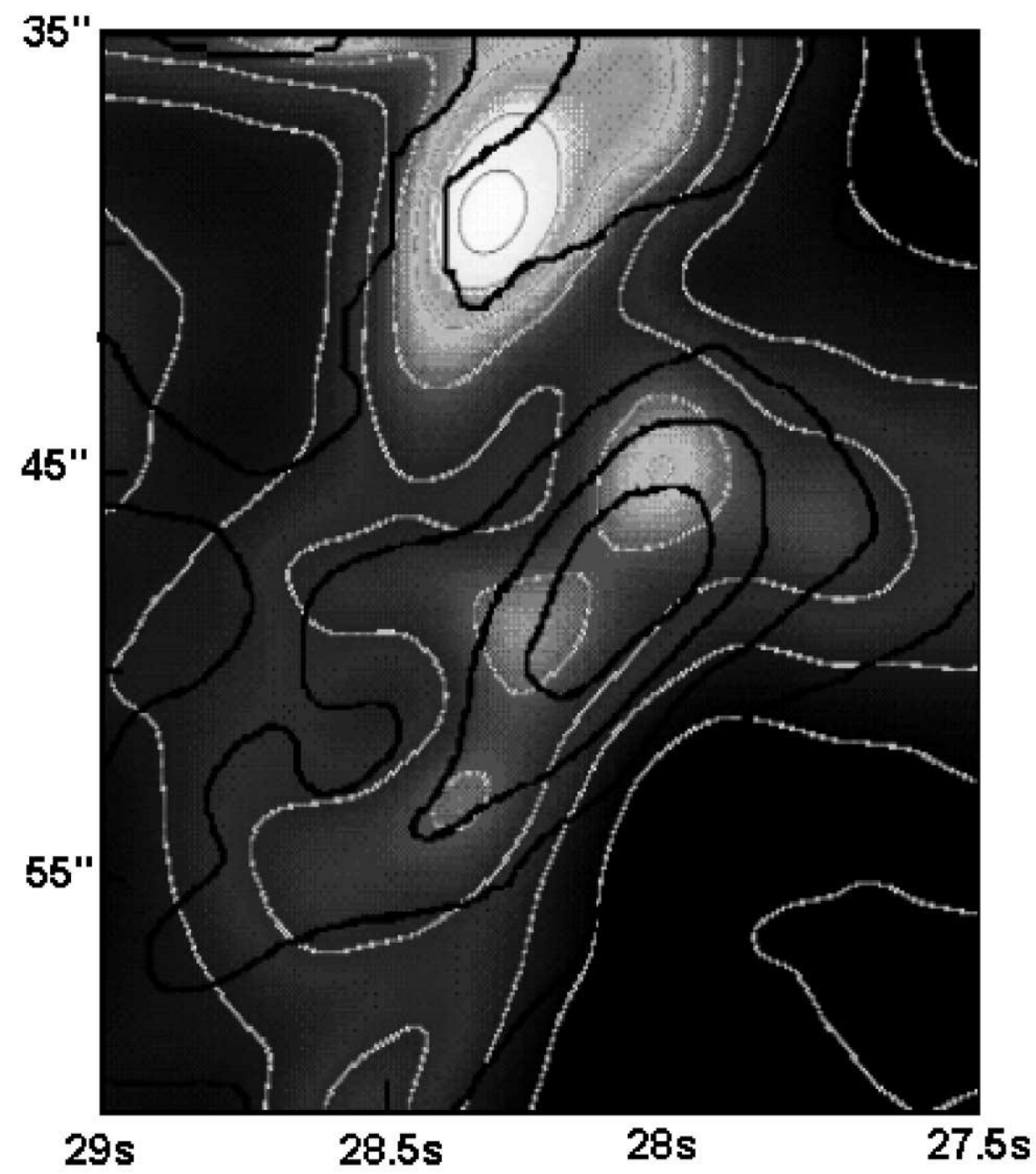

FIG. 5. - 8.3 GHz continuum emission contour map (dark contours) by Roberts et al. (1991) overlaid on our $10.8 \mu \mathrm{m}$ map

contour map overlaid on the optical depth map. Some of these temperature peaks are also coincident with brightness peaks, as shown in Figure $4 b$. We interpret the temperature enhancement and dust depletion in these areas as the result of embedded local sources clearing most of the material around them, while heating the remaining dust and ionizing the gas. Indeed, overlaying the $\mathrm{H} 92 \alpha(8.3 \mathrm{GHz})$ radio continuum map (2".4 angular resolution) by Roberts et al. (1991) on our $10.8 \mu \mathrm{m}$ map (Fig. 5) shows that the midinfrared emission follows very closely the distribution of the ionized gas, as previously indicated at lower resolution (Telesco et al. 1996).

\section{HEATING SOURCES IN THE CIRCUMNUCLEAR RING}

An embedded source heats the surrounding material, which reradiates at longer wavelengths. By assuming that our observed flux density is emitted by a blackbody the spectrum of which peaks at our observed wavelength, we can estimate a minimum luminosity for the heating sources in our maps. The flux densities from the brightest peak in our observations located at $0^{\prime \prime}, 8^{\prime \prime}$ in Figure 2 (source 1 from now on) at $10.8,11.7$, and $18.2 \mu \mathrm{m}$ in a $4^{\prime \prime}$ aperture radius are 3.7, 3.2, and $15.4 \mathrm{Jy}$, respectively. Using the flux density from the $18.2 \mu \mathrm{m}$ image and assuming a distance to the Galactic center of $8.5 \mathrm{kpc}$ and a $\tau_{\text {ISM }}$ at $18.2 \mu \mathrm{m}$ of 2.2 , we obtained a minimum luminosity for the source of $2000 L_{\odot}$. This lower limit corresponds to the luminosity of a B5 mainsequence star. We repeated this analysis for the infrared source located at $5^{\prime \prime}, 1^{\prime \prime}$ in the same figure (source 2), using an aperture radius of $3^{\prime \prime}$, and obtained a maximum flux density of $3.6 \mathrm{Jy}$ at $18.2 \mu \mathrm{m}$, which gives a minimum luminosity of $500 L_{\odot}$, corresponding to an B8 main-sequence star.

To further constrain the luminosity limits for these sources, we considered the Ne II continuum map of the Galactic center (Fig. 6) obtained by Lacy et al. (1991). Ne II emission traces the number of ionizing photons with little dependence on electron temperature and can be used to estimate the luminosity of the ionizing source. Following the analysis by Lacy, Beck, \& Geballe (1982) and Rubin (1968) and assuming solar abundances, an electron temperature of $10^{4} \mathrm{~K}$, and a distance to the sources of $8.5 \mathrm{kpc}$, we can use the following relation to estimate the number of Lyman continuum photons, $N_{\text {Lyc }}\left(\right.$ photons s $\left.{ }^{-1}\right) \simeq 3.5 \times 10^{65} F[\mathrm{Ne}$ II $]$. Here $F$ is the line flux in $\mathrm{W} \mathrm{cm}^{-2}$. The values obtained for sources 1 and 2 


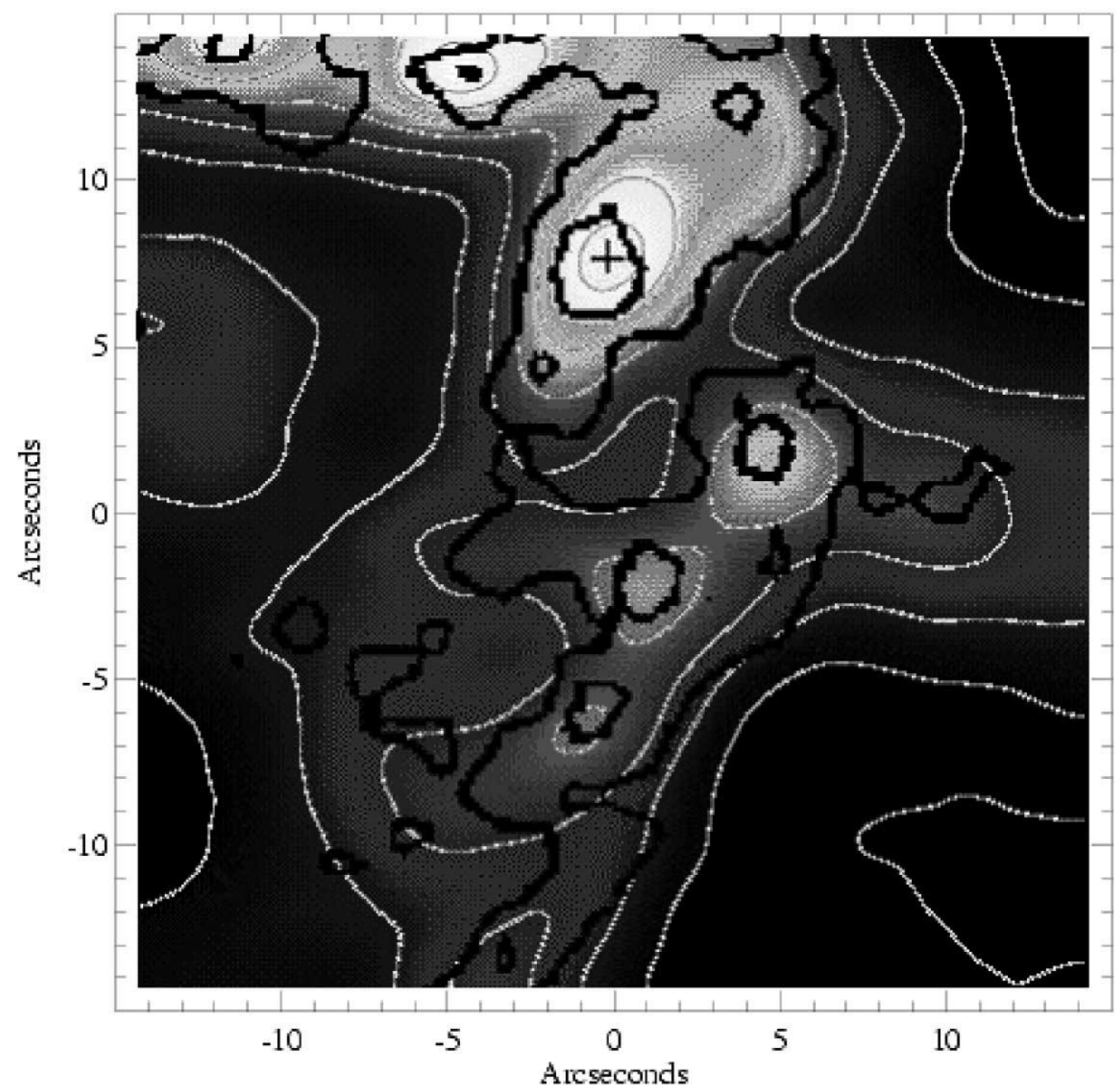

FIG. 6.-Ne II continuum map (dark contours) by Lacy et al. (1991) overlaid on our $10.8 \mu \mathrm{m}$ map

were $4.16 \times 10^{47}$ and $1.26 \times 10^{47}$ photons $\mathrm{s}^{-1}$, respectively. These values are still lower limits, since a fraction of the available ionizing photons from the central source could be absorbed or scattered by dust. If the ionizing sources are single main-sequence stars, these Lyman continuum photon rates for sources 1 and 2 correspond to stars of spectral types B0-B0.5 (Panagia 1973). The higher luminosity values obtained from the number of ionizing photons compared with the derived mid-infrared luminosities imply that these sources should have high far-infrared luminosities, and future observations with SOFIA might be able to resolve them.

Other heating sources in the central cluster have been identified as $\mathrm{He}$ I blue supergiants and less massive O9-B0.5 stars (Eckart, Thomas, \& Reinhard 1999). Our observations suggest that, if single sources are responsible for the observed luminosities, massive stars may also provide some local heating in the circumnuclear ring. The stars may have formed in the central cluster and migrated 1 to $2 \mathrm{pc}$ out to the CNR; a star moving at $20 \mathrm{~km} \mathrm{~s}^{-1}$ can travel $2 \mathrm{pc}$ in only $10^{5} \mathrm{yr}$, comparable to the lifetime of the most massive stars.
Another possibility is that the stars formed in the same cluster as the He I stars but outside the central few parsecs, as suggested by Gerhard (2001). In that scenario the cluster spiraled into the center of the Galaxy and more massive stars ended up at lower radii than their lower mass counterparts, which would now be just outside the central parsec of the Galactic center.

Regardless of the particular nature of these sources, it now at least seems evident that the CNR is locally heated by relatively young stars. The high resolution of mid-infrared detectors on new large telescopes, such as Gemini, VLT, and the GTC, will permit the detailed study of these sources in order to clarify such issues as source multiplicity and geometry.

We wish to thank the staff at IRTF for their excellent support and University of Florida engineers Kevin Hanna and Jeff Julian for their outstanding contribution to the OSCIR program. N. Mariñas also wants to thank the Minority Fellowship Program at the University of Florida. This research was partly supported by NSF grant AST 96-18348 to the University of Florida. 


\section{REFERENCES}

Aitken, D. K., Allen, M. C., \& Roche, P. F. 1982, in AIP Conf. Proc. 83 , The Galactic Center (Melville, NY: AIP), 67

Chan, K.-W., Moseley, S. H., Casey, S., Harrington, J. P., Dwek, E. J., Loewenstein, R., Varosi, F. \& Glaccum, W. 1997, ApJ, 483, 798

Eckart, A., Thomas, O., \& Reinhard, G. 1999, A\&A, 352, L22

Gatley, I. 1982, in AIP Conf. Proc. 83, The Galactic Center (Melville, NY: AIP), 25

Gerhard, O. 2001, ApJ, 546, L39

Gezari, D. Y., Tresch-Fienberg, R., Fazio, G. G., Hoffmann, W. F., Gatley, I., Lamb, G., Shu, P., \& McCreight, C. 1985, ApJ, 299, 1007

Gezari, D. Y., Dwek, E., \& Varosi, F. 1994, in The Nuclei of Normal Galaxies, ed. R. Genzel \& A. I. Harris (Dordrecht: Kluwer), 343

Krabbe, A., Genzel, R., Frapatz, S., \& Rotaciuc, V. 1991, ApJ, 382, L19

Lacy, J. H., Achtermann, J. M., \& Serabyn, E. 1991, ApJ, 380, L71
Lacy, J. H., Beck, S. C., \& Geballe, T. R. 1982, ApJ, 255, 510

Latvakoski, H. M., Stacey, G. J., Gull, G. E., \& Hayward, T. L. 1999, ApJ, 511,761

Mathis, J. S. 1990, ARA\&A, 28, 37

McCarthy, J. F., Forrest, W. J., Briotta, D. A., Jr., \& Houck, J. R. 1980, ApJ, 242, 965

Panagia, N. 1973, AJ, 78, 929

Rieke, G. H., \& Lebofsky, M. J. 1982, in AIP Conf. Proc. 83, The Galactic Center (Melville, NY: AIP), 194

Rieke, G. H., Telesco, C. M., \& Harper, D. A. 1978, ApJ, 220, 556

Roberts, D. A., Goss, W. M., van Gorkom, J. H., \& Hayward, R. L. 1991, ApJ, 366, L15

Rubin, R. H. 1968, ApJ, 154, 391

Telesco, C. M., Davidson, J. A., \& Werner, M. W. 1996, ApJ, 456, 541 